\title{
OPEN Asthma in paediatric intensive care in England residents: observational study
}

\author{
Mome Mukherjee ${ }^{1,2 \bowtie}$, Steve Cunningham ${ }^{1,3}$, Mohammad Romel Bhuia ${ }^{1}$, Tsz-Yan Milly Lo ${ }^{4}$, \\ Jasper V. Been ${ }^{1,5,6}$ \& Aziz Sheikh ${ }^{1,2}$
}

Despite high prevalence of asthma in children in the UK, there were no prior report on asthma admissions in paediatric intensive care units (PICU). We investigated the epidemiology and healthcare resource utilisation in children with asthma presenting to PICUs in England. PICANet, a UK national PICU database, was queried for asthma as the primary reason for admission, of children resident in England from April 2006 until March 2013. There were 2195 admissions to PICU for a median stay of 1.4 days. $59 \%$ were males and $51 \%$ aged $0-4$ years. The fourth and fifth most deprived quintiles represented $61 \%$ (1329) admissions and 73\% (11) of the 15 deaths. Deaths were most frequent in $10-14$ years age $(n=11,73 \%)$, with no deaths in less than 5 years age. $38 \%$ of admissions $(828 / 2193)$ received invasive ventilation, which was more frequent with increasing deprivation (13\% (108/828) in least deprived to $31 \%(260 / 828)$ in most deprived) and with decreasing age (0-4-year-olds: $49 \%$, 409/828). This first multi-centre PICU study in England found that children from more deprived neighbourhoods represented the majority of asthma admissions, invasive ventilation and deaths in PICU. Children experiencing socioeconomic deprivation could benefit from enhanced asthma support in the community.

Asthma is the most common chronic disease in children, with the prevalence higher in children in the UK than in the rest of Europe ${ }^{1}$. Asthma in UK children is responsible for substantial morbidity, with 2.8 million school days lost annually ${ }^{2}$, and more than 25,000 children admitted annually with asthma attacks ${ }^{3}$. Children's asthma also directly impacts family members with $69 \%$ of parents or carers taking time off work and $13 \%$ give up their jobs completely to support their child's asthma care ${ }^{1}$.

In children admitted to hospital in England, geographical and socio-economic status (SES) account for differences in asthma presentation and outcomes ${ }^{4}$. A single-centre study in the USA, which compared 161 paediatric intensive care units (PICU) to 610 non-PICU hospital admissions, reported that SES was not associated to PICU admission ${ }^{5}$. However, it was identified that among parents of the PICU admissions, $34 \%$ earned less than $\$ 15,000$, $45 \%$ were educated up to high school level, $40 \%$ could not find work despite seeking employment, $38 \%$ had no resource for borrowing money in time of need, $74 \%$ did not own a home and $25 \%$ did not own a car.

Admissions to paediatric intensive care with acute asthma are uncommon but may be considered a failure of asthma management and thus a bellwether of the general management of asthma in each community. A better understanding of the epidemiology and healthcare utilisation of children with acute asthma admitted to PICU may inform strategies to help reduce future asthma attack severity and mortality.

We describe the epidemiology and healthcare resource utilisation in children resident in England with asthma, who required admission to PICU.

\footnotetext{
${ }^{1}$ Asthma UK Centre for Applied Research, Usher Institute, The University of Edinburgh, Edinburgh, UK. ${ }^{2} \mathrm{HDR}$ UK Better Care and BREATHE Hub, The University of Edinburgh, Edinburgh, UK. ${ }^{3}$ Centre for Inflammation Research, Royal Hospital for Children and Young People, The University of Edinburgh, Edinburgh, UK. ${ }^{4}$ Paediatric Critical Care Unit, Royal Hospital for Children and Young People, Edinburgh, UK. ${ }^{5}$ Division of Neonatology, Department of Paediatrics, Department of Obstetrics and Gynaecology, and Department of Public Health, Erasmus MC-Sophia Children's Hospital, University Medical Centre Rotterdam, Rotterdam, The Netherlands. ${ }^{6}$ Division of General Internal Medicine and Primary Care, Brigham and Women's Hospital/Harvard Medical School, Boston, MA, USA. ${ }^{\boxplus e m a i l: ~ m o m e . m u k h e r j e e @ e d . a c . u k ~}$
} 


\section{Methods}

We interrogated electronic data collected prospectively between 1 April 2006 and 31 March 2013 of all PICU admissions of children resident in England, aged 0-14 years, who were given a primary diagnosis of asthma. The data includes children who had more than one admission in PICU for asthma. A protocol with a brief overview of methods has been published previously ${ }^{6}$. Asthma PICU admissions in England were compared to those in the whole of the UK and asthma deaths in PICU were compared to total deaths due to asthma as an underlying condition in England. We are reporting the study findings here following the REporting of studies Conducted using Observational Routinely-collected Data (RECORD) guideline ${ }^{7}$.

Database. In the UK, the Paediatric Intensive Care Audit Network (PICANet) ${ }^{8}$, is a dedicated PICU database which enables common audit across sites. The database receives core government funding to support its use $^{9}$. All UK PICU together with PICU in Ireland contribute data to PICANet ${ }^{8}$. We report results for England only due the small number of PICU admissions in Scotland, Wales and Northern Ireland. This means that information by SES was not available due to data confidentiality and that complete data over the study period was not available for these countries as they joined PICANet later. Since the data were originally obtained to estimate the cost of asthma to the public sector by financial year (i.e. 1 April to 31 March of next year) ${ }^{6}$, we included all National Health Service (NHS) PICU in England (excluding two private PICU) and retained the reporting convention by financial year, though PICANet data are reported annually from 1 January to 31 December each year. The number of English PICU contributing data varied over the study period due to addition of new PICU or merging of existing PICU. On average there were 25 PICU per year contributing to PICANet during the study period.

Some children who cannot be managed within the PICU of their resident UK-country receive care in PICU of another UK-country. Thus, we considered children whose country of residence was England even though they may have been treated elsewhere. Each child admitted to a participating PICU centre, provided information to the standardised PICANet dataset, including demographics, diagnosis, disease severity and clinical outcomes. Based on the findings recorded by intensivists, severity of illness in the first $24 \mathrm{~h}$ following PICU admission was auto-calculated based on an algorithm in the PICU electronic health record system, the 'paediatric index of mortality' score version 2 (PIM2), which was used in PICANet to standardise disease severity/care burden and to predict mortality in PICU ${ }^{10}$. The PIM2 mortality prediction model was an improvement of PIM version 1 and was developed using data at admission from 14 PICU in Australia, UK and New Zealand, using ten variables: (i) systolic blood pressure, (ii) pupillary reactions to bright light, (iii) partial pressure of oxygen $(\mathrm{PaO} 2)(>3 \mathrm{~mm}$ and both fixed $=1$, other or unknown $=0$ ), (iv) base excess in arterial or capillary blood, (v) mechanical ventilation at any time during the first hour in ICU $($ no $=0$, yes $=1)$, (vi) elective admission to ICU (no $=0$, yes $=1$ ), (vii) recovery from surgery or a procedure is the main reason for ICU admission (no =0, yes $=1$ ), (viii) admitted following cardiac bypass (no $=0$, yes $=1$ ), (ix) high risk diagnosis-none/cardiomyopathy or myocarditis/ cardiac arrest preceding ICU admission/hypoplastic left heart syndrome/severe combined immune deficiency/ HIV infection/leukaemia or lymphoma after first induction/liver failure/spontaneous cerebral haemorrhage/ neuro-degenerative disorder and $\mathrm{x}$ ) low risk diagnosis - none or main reason for PICU admission was asthma/ bronchiolitis/obstructive sleep apnoea/diabetic keto-acidosis ${ }^{10}$. PIM2 values ranged between 0 and $100 \%$, where $0 \%$ meant no risk of death and $100 \%$ meant absolute risk of death. Neonatal ICU or high dependency care admissions do not form part of the PICANet dataset.

The SES of children admitted to PICU in England was derived by PICANet by linking the validated home address via the National Statistics Postcode Directory ${ }^{11}$, which has information on the English Index of Multiple Deprivation (EIMD). We used the most recent EIMD (2010) for our data in our analysis as we wanted to compare SES over time. EIMD 2010 was constructed based on weighted deprivation in each of the 38 indicators, which were then categorised into seven domains of income, employment, health, education, housing, crime and living environment, in each of the 3248 geographical areas in England, which are designed to be of a similar population size with an average of approximately 1500 or 650 households ${ }^{12}$. SES was categorised into quintiles: EIMD quintile 1 (EIMD1) represented the least deprived and EIMD5 the most deprived neighbourhood.

Deaths statistics for national and local regions published by the Office for National Statistics (ONS) were used to find all asthma deaths in England, in children aged 0-14 years, during the study period ${ }^{13}$.

Study population and period. Only children who were aged under 15 years on the day of admission to PICU were included. Admissions with a primary diagnosis of asthma identified using Read codes version 3, published previously, were studied ${ }^{6}$.

Data and analyses. Information are provided for financial years, by 5-year age-groups (0-4, 5-9 and 10-14 years), sex and socio-economic status. Healthcare resource utilisation was measured by ventilation types (invasive ventilation only, non-invasive ventilation only, or having received both invasive and non-invasive ventilation) and length of stay. Any form of ventilation support includes admissions which had invasive ventilation only' as well as 'both invasive and non-invasive ventilation'. Outcome at PICU discharge was examined by survival. Number of deaths in PICU were compared to overall deaths for asthma, as an underlying condition, in under 15-year-olds in England.

Categorical data (age, sex, year, EIMD) were reported by counts and percentages, and continuous data (PIM2 and LoS) by median and inter-quartile range (IQR). The number of PICU admissions were age standardised using the English Standard Population. The denominators used were the respective mid-year population estimates for those age groups in June of that year. Age standardised rates and 95\% confidence intervals (CI) with Poisson approximation $^{14}$, were calculated per one million $(1,000,000)$ population. The one-way ANOVA test was used to 


\begin{tabular}{|c|c|c|c|c|c|c|c|c|}
\hline \multicolumn{2}{|c|}{$\begin{array}{l}\text { Asthma } \\
\text { admissions }\end{array}$} & \multicolumn{2}{|c|}{$0-4$ years } & \multicolumn{2}{|c|}{ 5-9 years } & \multicolumn{2}{|c|}{ 10-14 years } & \multirow{2}{*}{$\begin{array}{l}\text { Age-standardised rate per million } \\
(95 \% \mathrm{CI})\end{array}$} \\
\hline & $\mathbf{n}$ & $\%$ & Male \% & $\%$ & Male \% & $\%$ & Male \% & \\
\hline $2006-07$ & 266 & 45.5 & 66.9 & 28.6 & 56.5 & 25.9 & 51.3 & $28.9(28.4-29.5)$ \\
\hline $2007-08$ & 296 & 49.3 & 59.6 & 26.4 & 55.6 & 24.3 & 47.4 & $31.9(31.3-32.4)$ \\
\hline $2008-09$ & 328 & 55.2 & 65.2 & 19.8 & 56.1 & 25.0 & 47.7 & $34.8(34.2-35.4)$ \\
\hline $2009-10$ & 326 & 54.9 & 63.7 & 17.2 & 56.0 & 27.9 & 48.2 & $34.3(33.7-34.9)$ \\
\hline $2010-11$ & 327 & 54.4 & 60.7 & 20.5 & 63.4 & 25.1 & 56.7 & $34.0(33.4-34.6)$ \\
\hline $2011-12$ & 283 & 45.9 & 60.0 & 24.4 & 58.3 & 29.7 & 59.4 & $29.6(29.0-30.1)$ \\
\hline $2012-13$ & 369 & 52.6 & 55.2 & 23.0 & 57.8 & 24.4 & 60.0 & $37.6(37.0-38.2)$ \\
\hline Overall & 2195 & 51.1 & 61.6 & 22.8 & 57.7 & 26.0 & 53.0 & \\
\hline
\end{tabular}

Table 1. Admissions with asthma as the primary reason for admission in PICU in England with percentages by age-groups and males therein and age-standardised rate per million [number (n), percentages (\%) and 95\% confidence interval $(95 \% \mathrm{CI})]$.

find if the average number of admissions were same in the EIMD quintiles. Since PIM2 and LoS were skewed, non-parametric tests of comparison were used for them. Differences in PIM2 and LoS for age-group, EIMD and year was performed using Kruskal-Wallis Chi-square test ( $\mathrm{H}$ statistic, $p$ value). Differences in PIM2 and LoS for sex was performed using the Mann-Whitney $U$ test and Mantel-Haenszel Chi-square test for independence of ordinal categorical variables (EIMD, year). To find association of age, sex, EIMDs and year with PIM2 and LoS, separately, which were both positively skewed, age, sex and EIMDs were adjusted in regression models and finally a generalised linear model with gamma distribution and log link function was used, since it had the least Akaike Information Criterion. Multinomial logistic regression was used to find association between EIMDs and invasive ventilation, controlling for age, sex and year. We did not try to find factors that affected mortality, since we had a minimum dataset and did not have all the measurements taken in PICU. We only had years as a time measure, thus could not look at seasonality. Data were analysed using IBM SPSS Statistics version 25.

Ethics. PICANet has approvals to collect patient-identifiable data without informed consent from Patient Information Advisory Group (now the NHS Health Research Authority Confidentiality Advisory Group) and ethics approval granted by the Trent Medical Research Ethics Committee, ref. 05/MRE04/17+5. Ethics approval for anonymised patient level data access was obtained from PICANet and from the University of Edinburgh's Usher Institute's Ethics Review Group ${ }^{6}$.

\section{Results}

In the reporting period, 1 April 2006 to 31 March 2013, there were 2195 PICU admissions for asthma in children under 15 years of age resident in England. There were 78,615 children, including 15 years and above, admitted during the study period, of which 2110 children were for asthma (2.7\%).

Epidemiology. PICU admissions with a primary diagnosis of asthma are described in Table 1. The highest proportion of admissions were in those aged $0-4$ years $(51.1 \%, 1129 / 2195)$ with a male predominance $(58.6 \%$, $1286 / 2195)$. There was significantly higher risk of PICU admission for asthma in the $0-4$-year-olds compared to the 5-9 and 10-14-year-olds (t statistics $7.5(p<0.001)$ and $6.2(p<0.001)$ respectively) and in the 5-9-year-olds compared to the 10-14-year-olds ( $\mathrm{t}$ statistics $-2.2(p=0.02)$ ). Only in the $0-4$-year-olds, males had a significantly higher risk of PICU admission for asthma than females (t statistics $4.4(p=0.0004)$ ).

The estimated number of age standardised PICU admissions per million children in England, were 28.9 (95\% CI 28.4-29.5) in 2006-07 and 37.6 (95\% CI 37.0-38.2) in 2012-2013. Although the trend was positive $(\beta=0.74)$, it was not statistically significant.

Severity. The PIM2 score was assessed as a marker of disease severity. PIM2 was less than $1 \%$ in $72.5 \%$ admissions and was over $30 \%$ in $0.4 \%$ admissions. Median PIM2 were similar in both girls and boys over the study period [median $0.4 \%$ (IQR $0.2-1.0 \%)$ vs $0.4 \%$ (IQR $0.2-1.1 \%)(\mathrm{H}=1.67, p=0.196)$ ]. The overall trend in PIM2 significantly decreased [4.4\% (95\% CI 2.3-6.5\%; $p=0.000052)]$ when age, sex and EIMD were controlled (Supplementary Table S1, S2).

Compared to 0-4-year-olds, PIM2 when controlled for year and EIMD, was 1.13 times (95\% CI 1.01-1.26, $p=0.031)$ significantly higher in 5-9-year-olds and 1.95 times (95\% CI 1.74-2.19, $p<0.00001)$ significantly higher in 10-14-year-olds, indicating greater severity with increasing age (Supplementary Table S2, S3).

Healthcare resource utilisation. Mechanical ventilation data was missing for two admissions $(\mathrm{N}=2193)$ and LoS was missing in three admissions $(\mathrm{N}=2192)$. These admissions are reported in overall numbers but excluded from mechanical ventilation and LoS analyses.

Table 2 shows the distribution of ventilation in asthma admissions in PICU in England.

Over one-third of all admissions to PICU with asthma were mechanically ventilated $(37.8 \%, 828 / 2193)$. A further 35.8\% (95\% CI 35.5-36.2; 786/2193) admissions received only invasive ventilation, while $1.9 \%$ (95\% CI 


\begin{tabular}{|c|c|c|c|c|c|c|c|c|c|}
\hline & \multicolumn{2}{|c|}{$\begin{array}{l}\text { No } \\
\text { ventilation }\end{array}$} & \multicolumn{2}{|c|}{$\begin{array}{l}\text { Only non- } \\
\text { invasive } \\
\text { ventilation }\end{array}$} & \multicolumn{2}{|c|}{$\begin{array}{l}\text { Only } \\
\text { invasive } \\
\text { ventilation }\end{array}$} & \multicolumn{2}{|c|}{\begin{tabular}{|l|} 
Received \\
both \\
invasive and \\
non-invasive \\
ventilation
\end{tabular}} & \multirow[b]{2}{*}{ Denominator } \\
\hline & $\mathbf{n}$ & $\%$ & $\mathbf{n}$ & $\%$ & $\mathrm{n}$ & $\%$ & n & $\%$ & \\
\hline $2006-07$ & 149 & 56.2 & 8 & 3.0 & 101 & 38.1 & 7 & 2.6 & 265 \\
\hline $2007-08$ & 174 & 59.0 & 12 & 4.1 & 101 & 34.2 & 8 & 2.7 & 295 \\
\hline 2008-09 & 196 & 59.8 & 12 & 3.7 & 114 & 34.8 & 6 & 1.8 & 328 \\
\hline $2009-10$ & 208 & 63.8 & * & * & 103 & 31.6 & * & * & 326 \\
\hline $2010-11$ & 201 & 61.5 & * & * & 114 & 34.9 & * & * & 327 \\
\hline $2011-12$ & 157 & 55.5 & 8 & 2.8 & 112 & 39.6 & 6 & 2.1 & 283 \\
\hline $2012-13$ & 207 & 56.1 & 14 & 3.8 & 141 & 38.2 & 7 & 1.9 & 369 \\
\hline Overall & 1292 & 58.9 & 73 & 3.3 & 786 & 35.8 & 42 & 1.9 & 2193 \\
\hline
\end{tabular}

Table 2. Number and percentages of paediatric admissions in England in the financial years in PICANet by ventilation provided. ${ }^{*}$ One of those counts were less than 5 , hence four are starred to prevent disclosure.

\begin{tabular}{|c|c|c|c|c|c|}
\hline & Number of admissions (\%) & PIM2\% (median, IQR) & $\begin{array}{l}\text { Any form of ventilation support (\%, } \\
95 \% \mathrm{CI})\end{array}$ & Length of stay (median, IQR) & Deaths [n (\%)] \\
\hline EIMD1 least deprived & $249(11.3)$ & $0.5(0.2-1.2)$ & $108(13.0(10.7-15.3))$ & $1.6(0.8-2.5)$ & $2(13.3(11.0-15.7))$ \\
\hline EIMD2 & $251(11.4)$ & $0.6(0.3-1.2)$ & $117(14.1(11.8-16.5))$ & $1.6(0.9-2.6)$ & $1(6.7(5.0-8.4))$ \\
\hline EIMD3 & $366(16.7)$ & $0.4(0.2-1.0)$ & $139(16.8(14.2-19.3))$ & $1.5(0.8-2.6)$ & $1(6.7(5.0-8.4))$ \\
\hline EIMD4 & $505(23.0)$ & $0.5(0.2-1.1)$ & $20424.6((21.7-27.6))$ & $1.5(0.8-2.8)$ & $3(20.0(17.3-22.7))$ \\
\hline EIMD5 most deprived & $824(37.5)$ & $0.4(0.2-1.0)$ & $260(31.4(28.2-34.6))$ & $1.3(0.7-2.4)$ & $8(53.3(49.9-56.7))$ \\
\hline Overall & $2195(100.0)$ & $0.4(0.2-1.1)$ & $828(100.0)$ & $1.4(0.8-2.6)$ & $15(100)$ \\
\hline
\end{tabular}

Table 3. Severity and outcomes of PICU admissions with asthma as primary diagnosis or as underlying condition for death, by socio-economic status in England during 2006-2007 to 2012-2013.

$1.9-2.0 ; 42 / 2193)$ received both invasive and non-invasive ventilatory support. Tracheostomy was performed in $0.7 \%$ of admissions. Any form of ventilation support was more commonly provided to 0-4-year-olds (49.4 (95\% CI 46.0-52.8), 409/828), in males (62.3 (95\% CI 59.0-65.6), 516/828) (Supplementary Table S3) and no difference was found over time (years).

Length of stay in PICU: Median LoS was 1.4 days (IQR 0.8-2.6) and remained much the same over the study period, when controlled for age, sex and EIMD $(\mathrm{p}=0.31)$ (Supplementary Tables S1, S4). LoS was similar across sex and age groups: males median 1.4 (IQR 0.8-2.6), females $1.5(0.9-2.6), 0-4$ years $1.4(0.8-2.6), 5-9$ years 1.4 (0.8-2.8), 10-14 years $1.3(0.8-2.5)$ days (Supplementary Table S3).

Socioeconomic status. The number of asthma PICU admissions increased with increase in deprivation (Table 3), which was irrespective of age $(\mathrm{F}=37.2, p<0.001)$.

After adjusting for age, sex and year, PIM2 score was 1.28 times higher (95\% CI 1.10-1.49) in the least deprived areas, compared to those in the most deprived areas. Any form of ventilation support increased with increasing levels of deprivation ( $p=0.000423$ ): $13.0 \%$ in least deprived to $31.4 \%$ in most deprived (Table 3 ). In the multinomial regression, it was found that any form of ventilation, compared to no ventilation support was significantly less in females than in males $(p=0.007)$, in 0 -4-year-olds $(p=0.01)$ compared to 10 -14-year-olds and in most to least deprived areas $(p=0.001)$ (Supplementary Table S4).

LoS was not significantly different across SES in England (Supplementary Table S5): 1.3 vs 1.6 median days in the most deprived versus the least deprived (Table 3). After adjusting for year and EIMD, LoS was 1.14 times (95\% CI 1.04-1.25, $p=0.006)$ higher in 10-14-year-olds compared to 0 -4-year-olds and $9 \%$ lesser in males than in females (95\% CI 2-15, $\mathrm{p}=0.017)$ (Supplementary Table S5).

Survival. 15 deaths occurred in children admitted to PICU with a primary diagnosis of asthma, with the majority in the $10-14$ years olds group $(73 \%, 11 / 15)$ and none in the pre-school group (Supplementary Table S3). Mortality rate was $0.7 \%(15 / 2195)$. Median PIM2 was higher amongst the non-survivors (31.7\%, IQR 2.6-95.3\%) compared to the survivors $(0.4 \%$, IQR $0.2-42.3 \%)$. Of the 15 who died, 14 (93.3\%) required invasive ventilation. The one child who died without any ventilation support was in PICU for less than an hour. Children who died had a longer LoS than the survivors [median 2.9 (1.9-11.4) vs. $1.4(0.8-23.8)$ days]. $60.7 \%$ admissions from the deprived neighbourhoods (EIMD 4,5) contributed to 73\% deaths (11/15) (Table 3). From ONS records, there were 127 deaths (as the underlying cause) in England, in under 15-year-olds, from asthma in the seven calendar years during 2006-2012. We found in the financial years 2006-2013, around 11.8\% (15/127) of them were in PICU. 


\section{Discussion}

This is the first study profiling the epidemiology, healthcare resource utilisation and outcomes of asthma as the primary condition in children resident in England admitted in PICU, found that children from deprived neighbourhoods (EIMD 4,5$)$ comprised most of the admissions $(61 \%)$, received mechanical ventilation most frequently (60\%) and suffered the highest proportion of deaths (75\%). Deaths were more common in 10-14-yearolds. PICU admission for asthma is infrequent and there was no clear trend. Most admissions and mechanical ventilation occur in preschool children. Deaths in PICU represented only $12 \%$ of all deaths due to asthma over the years investigated.

The PICANet dataset used here is of high quality from a national audit network on PICU in the UK, with high data completeness for England. Sample selection bias is minimised because PICANet covers the entire childhood population admitted to PICU in England. Limitations of the study were: (i) asthma is often difficult to diagnose in children under five years of age. It is quite probable that admissions coded as asthma represented other acute wheezing conditions, including acute viral induced wheeze ${ }^{15,16}$. Preschool admissions with a diagnosis of asthma were higher than those reported from other countries ${ }^{5,17-25}$. (ii) Due to confidentiality issues coupled with small numbers from Scotland, Wales and Northern Ireland or the PICU asthma deaths in England, detailed analyses from these episodes were not possible, thus limiting our ability to comment on reasons for regional variations or deaths ${ }^{26}$. (iii) PICANet dataset does not capture children admitted to adult ICU for asthma treatment. This number is considered small because children admitted into adult ICU have been referred to PICU in the UK since $1996^{27}$. (iv) The data of PICU admissions do not furnish us with the number of childhood patients. Thus, if a child had multiple admissions, that would inflate the association with SES. (v) Since the data were originally obtained to study the burden of asthma ${ }^{2}$, we did not collect clinical data such as arterial blood gases, blood cell count, chest $\mathrm{X}$ ray, lung function, etc. (vi) Asthma was a low-risk diagnosis in PIM2. We did not have access to predictive severity score which is asthma specific, for example the initial Modified Pulmonary Index Score $(\mathrm{MPIS})^{28}$. (vii) Our data pertained to all admissions during PICU stay only and did not include pre or posthospitalisations. Furthermore, some patients may have been admitted more than once in the year. Thus we cannot compare to findings where frequency and time to PICU re-admissions were found to be similar in Canada and USA and a higher proportion of children were readmitted to PICU in USA ${ }^{29}$.

Whilst asthma related PICU deaths in England (0.7\%) were similar to those found in Australia (0.6\%) and the Netherlands $(0.6 \%)^{17,18}$, the reporting of asthma mortality in multi-centre PICU studies in the USA varied $(0.3 \%, 1.9 \%, 4.3 \%)^{19,21,30}$. A UK study on general admissions in PICU using PICANet, found children who died in PICU stayed longer than who survived ${ }^{31}$, similar to our study. Given the small number of asthma deaths, we could not find if there was a reduction in asthma deaths in England's PICU over time. One UK study on all paediatric admissions in adult ICU reported that crude mortality fell from 6.7 in 1996 to $2.8 \%$ in 2011 (overall $4.6 \%)^{26}$. The reduction in PICU deaths was ascribed to development of PICU in the UK from its infancy in the nineties, whereby many children who would have been admitted to adult ICU by mid 2000s were admitted to PICU or referred promptly from adult ICU to PICU ${ }^{26}$. Thus the observed fall in percentage might not be a true trend. Most deaths from asthma in children in England are outside PICU (88\%), as also observed in Australia $(78 \%)$ and Ireland $(100 \%)^{32,33}$.

Our previous study had found that although prevalence of severe asthma and hospitalisations were higher in children in lower SES, asthma deaths in the community were higher in children from higher SES ${ }^{4}$ This study, however, finds that asthma deaths in PICU were higher in children from lower SES. Although it is difficult to find ascribe clear reasons for this anomaly, it could be speculated that there are some psychosocial processes that contribute to this ${ }^{34}$. Air pollution and parental stress were found to be associated to increased childhood asthma, which was more prevalent in children living in lower SES areas ${ }^{35}$. The National Review of Asthma Deaths (NRAD) had reported that two-third of the asthma deaths could be preventable if they were attentively managed in primary care ${ }^{3}$. Identifying children in whom a tailored asthma management may prevent PICU admission could target those with asthma symptoms for over a week, older and/or exposed to tobacco smoke ${ }^{36}$.

The other considerable consequence of a PICU admission is the emotional burden in individuals who needed intensive care and their parents and carers, irrespective of survival ${ }^{37-41}$. This, and the decline in pulmonary function after PICU discharge ${ }^{42}$, highlight that healthcare professionals need to understand the entire patient journey from asthma diagnosis, asthma exacerbation to discharge, so that patients are better managed and the need for a PICU admission does not arise ${ }^{43-45}$. Since asthma triggers and symptoms vary in individuals, it is important to consider the family in communications and decision making ${ }^{43-45}$.

Use of mechanical ventilation in children admitted to PICU in England (37.8\%) is higher than that found in most other multi-centre reports from Australia (6-14\%), the Netherlands (19\%), while it varied in USA (19\%, $3-47 \%)^{17,18,21,46}$. This may reflect a lower threshold for PICU admission in some countries, i.e. increasing the denominator. Unlike our study, a multi-centre study in the Netherlands found a trend for increasing mechanical ventilation use over time ${ }^{18}$, with an overall levels of half of those that we report (36\% vs. $\left.19 \%\right)^{47}$. The length of PICU stay they reported was double that we report (median 1.4 vs. 3 days) but with similar mortality rates $(0.7 \%$ vs. $0.6 \%)^{18}$, suggesting a lower threshold for admission. The male preponderance found in our study concurs with the literature ${ }^{5,17-24,46,48,49}$.

This is the first study utilising data from multiple sites to report the association of SES on asthma in PICU admissions. The population distribution of under-15-year-olds in England in 2010 was $24.0 \%$ in most deprived neighbourhoods and $19.3 \%$ in least deprived neighbourhoods. We found $37.5 \%$ admissions from most deprived neighbourhoods and $11.3 \%$ from least deprived neighbourhoods. Unlike a single-centre study in USA ${ }^{5}$, we did find association of deprivation to PICU admissions, length of stay and deaths. Furthermore, this is also the first study that found that asthma severity, as determined by adjusted PIM2 score, could be higher in children from higher SES. 
This study highlights an urgent need to identify ways to improve asthma outcomes for children, particularly in deprived communities. Addressing the causes of acute asthma exacerbations through education and appropriate treatment should lower PICU admissions. Ideally this should be delivered within primary care, especially in difficult to reach communities where access to primary care is limited.

In addition to clinical treatment, there remains an unmet need for novel therapeutics to alleviate severe cases of acute asthma exacerbations. Little is known about the efficacy of biologic therapies in paediatric asthma, their optimum duration for treatment and potential long term side effects into adulthood ${ }^{50-53}$. Thus the Global Initiative for Asthma (GINA) recently published considerations for selecting biologic therapy for severe asthma in children younger than 18 years is helpful ${ }^{54}$.

A focus on asthma education of children, parents and caregivers to act early in preventing acute asthma exacerbations from progressing should be a key objective to reduce both hospital and PICU admissions. Such education can be delivered across social and healthcare settings and needs to be repeated regularly to ensure good understanding and ability to respond when needed.

\section{Conclusions}

In this first study of data across multiple sites in England we found that there are over 300 PICU admissions in the critically ill children with asthma in a year and children from more deprived neighbourhoods had most of the asthma admissions, invasive ventilation and deaths in PICU. Reducing severe asthma attacks will require a coherent, consistent and cross-disciplinary approach (healthcare professionals, schools, third sector) to raise awareness of severe asthma and how children and parents/caregivers can reduce its impact.

\section{Data availability}

The data that support the findings of this study are available from PICANet (https://www.picanet.org.uk/datacollection/data-requests/) but restrictions apply to the availability of these data and so are not publicly available. Analysed data are presented in the paper and supplementary file.

Received: 10 August 2021; Accepted: 4 January 2022

Published online: 25 January 2022

\section{References}

1. Gibson GJ, Loddenkemper R, Sibille Y \& B, L. in Chapter: Childhood asthma (European Respiratory Society, 2013).

2. Mukherjee, M. et al. The epidemiology, healthcare and societal burden and costs of asthma in the UK and its member nations: Analyses of standalone and linked national databases. BMC Med. 14, 1-15. https://doi.org/10.1186/s12916-016-0657-8 (2016).

3. Levy, M. L. The national review of asthma deaths: What did we learn and what needs to change?. Breathe 11, 14-24. https://doi. org/10.1183/20734735.008914 (2015)

4. Gupta, R. P., Mukherjee, M., Sheikh, A. \& Strachan, D. P. Persistent variations in national asthma mortality, hospital admissions and prevalence by socioeconomic status and region in England. Thorax 73, 706-712. https://doi.org/10.1136/thoraxjnl-2017-210714 (2018).

5. McDowell, K. M., Kercsmar, C. M., Huang, B., Guilbert, T. W. \& Kahn, R. S. Medical and social determinants of health associated with intensive care admission for asthma in children. Ann. Am. Thorac. Soc. 13, 1081-1088. https://doi.org/10.1513/AnnalsATS. 201512-798OC (2016).

6. Mukherjee, M. et al. Estimating the incidence, prevalence and true cost of asthma in the UK: Secondary analysis of national standalone and linked databases in England, Northern Ireland, Scotland and Wales-A study protocol. BMJ Open. https://doi.org/10. 1136/bmjopen-2014-006647 (2014).

7. Benchimol, E. I. et al. The REporting of studies conducted using observational routinely-collected health data (RECORD) statement. PLoS Med. 12, e1001885. https://doi.org/10.1371/journal.pmed.1001885 (2015).

8. Paediatric Intensive Care Audit Network (PICANet). Paediatric Intensive Care Audit Network Annual Report 2012-2014 (published 2015): Universities of Leeds and Leicester. http://www.picanet.org.uk/Audit/Annual-Reporting/ (2015).

9. Department of Health-Health Service Directorate. Paediatric Intensive Care "A Framework for the Future" Report from the National Coordinating Group on Paediatric Intensive Care to The Chief Executive of the NHS Executive. http://www.ihrdni.org/315-016-1. pdf (1997)

10. Slater, A., Shann, F. \& Pearson, G. PIM2: A revised version of the Paediatric Index of Mortality. Intensive Care Med. 29, 278-285. https://doi.org/10.1007/s00134-002-1601-2 (2003).

11. Office of National Statistics (ONS). Postcode Directory (ONSPD). http://webarchive.nationalarchives.gov.uk/20160105160709/ http://www.ons.gov.uk/ons/guide-method/geography/products/postcode-directories/-nspp-/index.html (2016)

12. Department for Communities and Local Government. The English Indices of Deprivation 2010. https://www.gov.uk/government/ uploads/system/uploads/attachment_data/file/6871/1871208.pdf (2011).

13. Office for National Statistics (ONS). All data related to deaths. https://www.ons.gov.uk/peoplepopulationandcommunity/birth sdeathsandmarriages/deaths/datalist (2021).

14. Scottish Public Health Observatory (ScotPHO). Methodology. Standardisation Guidance. Calculation of standardised rates and ratios: Direct and indirect methods. https://www.scotpho.org.uk/media/1400/phi-standardisation-guidance-v21.docx (2021).

15. E. R. McFadden, J. Acute severe asthma. Am. J. Respir. Crit. Care Med. 168, 740-759. https://doi.org/10.1164/rccm.200208-902SO (2003).

16. Puranik, S., Forno, E., Bush, A. \& Celedón, J. C. Predicting severe asthma exacerbations in children. Am. J. Respir. Crit. Care Med. 195, 854-859. https://doi.org/10.1164/rccm.201606-1213PP (2017).

17. Rampersad, N., Wilkins, B. \& Egan, J. R. Outcomes of paediatric critical care asthma patients. J. Paediatr. Child Health https://doi. org/10.1111/jpc.13855 (2018).

18. Boeschoten, S. A. et al. Children with severe acute asthma admitted to Dutch PICUs: A changing landscape. Pediatr. Pulmonol. 53, 857-865. https://doi.org/10.1002/ppul.24009 (2018).

19. Shibata, S., Khemani, R. G. \& Markovitz, B. Patient origin is associated with duration of endotracheal intubation and PICU length of stay for children with status asthmaticus. J. Intensive Care Med. 29, 154-159. https://doi.org/10.1177/0885066613476446 (2014).

20. Sheikh, S., Khan, N., Ryan-Wenger, N. A. \& McCoy, K. S. Demographics, clinical course, and outcomes of children with status asthmaticus treated in a pediatric intensive care unit: 8-year review. J Asthma 50, 364-369. https://doi.org/10.3109/02770903.2012. 757781 (2013). 
21. Bratton, S. L. et al. Critical care for pediatric asthma: Wide care variability and challenges for study. Pediatr. Crit. Care Med. 13, 407-414. https://doi.org/10.1097/PCC.0b013e318238b428 (2012).

22. van den Bosch, G. E. et al. Risk factors for pediatric intensive care admission in children with acute asthma. Respir. Care 57, 1391-1397. https://doi.org/10.4187/respcare.01325 (2012).

23. Sala, K. A. et al. Factors associated with the development of severe asthma exacerbations in children. J. Asthma 48, 558-564. https:// doi.org/10.3109/02770903.2011.585411 (2011).

24. Al-Eyadhy, A. et al. Asthma changes at a pediatric intensive care unit after 10 years: Observational study. Ann. Thorac. Med. 10, 243-248. https://doi.org/10.4103/1817-1737.165302 (2015).

25. Chiang, B. L. et al. Clinical course and outcome of children with status asthmaticus treated in a pediatric intensive care unit: A 15-year review. J. Microbiol. Immunol. Infect. 42, 488-493 (2009).

26. Ramnarayan, P. et al. Effect of specialist retrieval teams on outcomes in children admitted to paediatric intensive care units in England and Wales: A retrospective cohort study. Lancet 376, 698-704. https://doi.org/10.1016/S0140-6736(10)61113-0 (2010).

27. Ramnarayan, P. et al. Characteristics and outcome of children admitted to adult intensive care units in England, Wales and Northern Ireland (1996-2011). Intensive Care Med. 39, 2020-2027. https://doi.org/10.1007/s00134-013-3010-0 (2013).

28. Miller, A. G. et al. Initial modified pulmonary index score predicts hospital length of stay for asthma subjects admitted to the pediatric intensive care unit. Respir. Care 65, 1227-1232. https://doi.org/10.4187/respcare.07396 (2020).

29. Tse, S. M. Asthma-related hospitalizations following critical asthma in children: A comparison between Canada and the United States. Respir. Med. 170, 106028. https://doi.org/10.1016/j.rmed.2020.106028 (2020).

30. Newth, C. J. et al. Fatal and near-fatal asthma in children: The critical care perspective. J. Pediatr. 161, 214-221.e213. https://doi. org/10.1016/j.jpeds.2012.02.041 (2012).

31. Plunkett, A. \& Parslow, R. C. Is it taking longer to die in paediatric intensive care in England and Wales?. Arch. Dis. Child. 101, 798-802. https://doi.org/10.1136/archdischild-2015-309592 (2016).

32. Robertson, C. F., Rubinfeld, A. R. \& Bowes, G. Pediatric asthma deaths in Victoria: The mild are at risk. Pediatr. Pulmonol. 13, 95-100. https://doi.org/10.1002/ppul.1950130207 (1992).

33. Richardson, S. C., O’Riordan, A. M., Linnane, B. \& Cox, D. W. Paediatric asthma deaths in Ireland: 2006-2016. Ir. J. Med. Sci. 189, 1337-1342. https://doi.org/10.1007/s11845-020-02234-2 (2020).

34. Marmot, M. Inequalities in asthma mortality: A specific case of a general issue of health inequalities. Thorax $73,704-705$. https:// doi.org/10.1136/thoraxjnl-2018-211573 (2018).

35. Deng, Q., Deng, L., Lu, C., Li, Y. \& Norbäck, D. Parental stress and air pollution increase childhood asthma in China. Environ. Res. 165, 23-31. https://doi.org/10.1016/j.envres.2018.04.003 (2018).

36. Boeschoten, S. A. et al. Risk factors for intensive care admission in children with severe acute asthma in the Netherlands: A prospective multicentre study. ERJ Open Res. 6, 00126-02020. https://doi.org/10.1183/23120541.00126-2020 (2020).

37. Rees, G., Gledhill, J., Garralda, M. E. \& Nadel, S. Psychiatric outcome following paediatric intensive care unit (PICU) admission: A cohort study. Intensive Care Med. 30, 1607-1614. https://doi.org/10.1007/s00134-004-2310-9 (2004)

38. Shudy, M. et al. Impact of pediatric critical illness and injury on families: A systematic literature review. Pediatrics 118(Suppl 3), S203-218. https://doi.org/10.1542/peds.2006-0951B (2006).

39. Rennick, J. E., St-Sauveur, I., Knox, A. M. \& Ruddy, M. Exploring the experiences of parent caregivers of children with chronic medical complexity during pediatric intensive care unit hospitalization: An interpretive descriptive study. BMC Pediatr. 19, 272. https://doi.org/10.1186/s12887-019-1634-0 (2019).

40. Logan, G. E., Sahrmann, J. M., Gu, H. \& Hartman, M. E. Parental mental health care after their child's pediatric intensive care hospitalization*. Pediatr. Crit. Care Med. 21, 941-948. https://doi.org/10.1097/pcc.0000000000002559 (2020).

41. Colville, G. The tip of an iceberg? A "big data" study examines the evidence for new mental health problems in parents after PICU. Pediatr. Crit. Care Med. 21, 1002-1003. https://doi.org/10.1097/pcc.0000000000002588 (2020).

42. Major, S., Vézina, K. \& Tse, S. M. Lung function of children following an intensive care unit admission for asthma. Pediatr. Allergy Immunol. Pulmonol. 34, 1-6. https://doi.org/10.1089/ped.2020.1271 (2021).

43. Brick, T. \& Parslow, R. C. Doing more of less: What registry data tell us about death in PICU. Intensive Care Med. 45, $1272-1274$. https://doi.org/10.1007/s00134-019-05714-x (2019).

44. Fraser, L. K., Bluebond-Langner, M. \& Ling, J. Advances and challenges in European paediatric palliative care. Medical sciences (Basel, Switzerland). https://doi.org/10.3390/medsci8020020 (2020).

45. ICUsteps. Supporting intensive care patients and relatives. https://www.icusteps.org/ (2005-2020).

46. Roberts, J. S., Bratton, S. L. \& Brogan, T. V. Acute severe asthma: Differences in therapies and outcomes among pediatric intensive care units. Crit. Care Med. 30, 581-585 (2002).

47. Hartman, M. E., Linde-Zwirble, W. T., Angus, D. C. \& Watson, R. S. Trends in admissions for pediatric status asthmaticus in New Jersey over a 15-year period. Pediatrics 126, e904-911. https://doi.org/10.1542/peds.2009-3239 (2010).

48. Paret, G. et al. Severe acute asthma in a community hospital pediatric intensive care unit: A ten years' experience. Ann. Allergy Asthma Immunol. 80, 339-344. https://doi.org/10.1016/s1081-1206(10)62980-8 (1998).

49. Abu-Ekteish, F. M., Zahraa, J. N., Al-Mobaireek, K. F., Nasir, A. A. \& Al-Frayh, A. S. The management of acute severe asthma in a pediatric intensive care unit. Saudi Med. J. 24, 388-390 (2003).

50. Saglani, S. et al. Biologics for paediatric severe asthma: Trick or TREAT?. Lancet Respir. Med. 7, 294-296. https://doi.org/10.1016/ s2213-2600(19)30045-1 (2019).

51. Golebski, K. et al. Expert meeting report: Towards a joint European roadmap to address the unmet needs and priorities of paediatric asthma patients on biologic therapy. ERJ Open Res. https://doi.org/10.1183/23120541.00381-2021 (2021).

52. Lovinsky-Desir, S. The use of biologic therapies for the management of pediatric asthma. Pediatr. Pulmonol. 55, 803-808. https:// doi.org/10.1002/ppul.24613 (2020).

53. Lozano, J., Nafria, B. \& Alsina, L. The long road to biologic therapies for asthma in pediatric patients. Arch. Bronconeumol. (Engl Ed) 57, 249-250. https://doi.org/10.1016/j.arbres.2020.08.003 (2021).

54. Global Initiative for Asthma (GINA). Difficult-to-treat and severe asthma in adolescent and adult patients. Diagnosis and management. A GINA pocket guide for health professionals. Version 3. (2021).

\section{Acknowledgements}

The grant holders were also Prof. Ceri J. Phillips, Swansea University, UK; Prof. David P. Strachan, St. George's, University of London, UK and Prof. Gwyneth A. Davies, Swansea University, UK. We are grateful to PICANet for their data, particularly to Melpo Kapetanstrataki, Dr. Sarah Fleming, Dr. Roger Parslow and Philip McShane; Shelley A. Boeschoten, Department of Paediatric Intensive Care, Erasmus Medical Centre, Sophia's Children Hospital, Rotterdam, The Netherlands for initial discussion; This work uses data provided by patients and collected by the NHS as part of their care and support. 


\title{
Author contributions
}

A.S. was the P.I. for the asthma burden study (https://doi.org/10.1186/s12916-016-0657-8). M.M. conceived the idea for this paper, analysed the data and wrote the drafts. All co-authors contributed to the work, reviewed and commented on the drafts. All authors read and approved the final manuscript.

\section{Funding}

This study was funded by Asthma UK (reference: AUK-PG-2012-178). MM's time to write the paper was supported by Health Data Research UK, which is funded by the UK Medical Research Council, Engineering and Physical Sciences Research Council, Economic and Social Research Council, Department of Health and Social Care (England), Chief Scientist Office of the Scottish Government Health and Social Care Directorates, Health and Social Care Research and Development Division (Welsh Government), Public Health Agency (Northern Ireland), British Heart Foundation and the Wellcome Trust. PICANet is funded by the National Clinical Audit and Patient Outcomes Programme, administered by the Healthcare Quality Improvement Partnership (HQIP); Welsh Health Specialised Services Committee; NHS Lothian/National Service Division NHS Scotland; the Royal Belfast Hospital for Sick Children; National Office of Clinical Audit Ireland (NOCA) and HCA International.

\section{Competing interests}

The authors declare no competing interests.

\section{Additional information}

Supplementary Information The online version contains supplementary material available at https://doi.org/ 10.1038/s41598-022-05414-5.

Correspondence and requests for materials should be addressed to M.M.

Reprints and permissions information is available at www.nature.com/reprints.

Publisher's note Springer Nature remains neutral with regard to jurisdictional claims in published maps and institutional affiliations.

\begin{abstract}
Open Access This article is licensed under a Creative Commons Attribution 4.0 International License, which permits use, sharing, adaptation, distribution and reproduction in any medium or format, as long as you give appropriate credit to the original author(s) and the source, provide a link to the Creative Commons licence, and indicate if changes were made. The images or other third party material in this article are included in the article's Creative Commons licence, unless indicated otherwise in a credit line to the material. If material is not included in the article's Creative Commons licence and your intended use is not permitted by statutory regulation or exceeds the permitted use, you will need to obtain permission directly from the copyright holder. To view a copy of this licence, visit http://creativecommons.org/licenses/by/4.0/.
\end{abstract}

(C) The Author(s) 2022 